tion, or to cause effusion into the orbital cellular texture; and toloss of tonicity in the orbital muscles, so that the globes, as it were, drop forwards. The last is, perhaps, the least likely of these unlikely things. There is not any loss of voluntary power, which I think would be inevitable, were there loss of tonicity on the orbital muscles; and the freest movement of the eyes may be combined with the greatest protrusion. Again, in the most debilitating diseases, with perfect muscular prostration, the eyeballs do not protrude.

I am inclined to attribute the protrusion to congestion of the deep-seated veins of the orbit, which I think offers a better explanation than any other of the variable amount of the exophthalmia, and of the readiness with which the eyeballs can be replaced by gentle pressure. Mr. Taylor, adopting Dr. Marshall Hall's views as to the spasmodic contraction of the muscles of the neck in paroxysmal and convulsive diseases, suggests that this may be the cause of the impeded return of the blood from the head; and this view is supported by the fact that, in the only two post mortem examinations that have been made, the internal jugular veins were found to be much dilated, as though there had long been some cause of obstruction at the lower part of their course; and, as in neither case was there any solid growth which could have impeded the circulation, it is not unreasonable to suppose that the obstacle was due to muscular spasm. But if this were true, how is it that there is no cerebral congestion, when the return of the blood from the brain is so checked?

It has been supposed that, if venous obstruction be the cause of the protrusion, to enlargement of the thyroid gland must the obstruction be attributed. The reader must decide how much this is worth, when he is told that considerable protrusion of the eyes is met with when there is not any perceptible thyroidal swelling.

From what has been said as to the nature of this disease, it will be obvious that the treatment must be directed towards overcoming the exciting cause of the anæmia, which, in the great majority of instances, depends upon uterine disorder. In addition to the special means which may be adopted for this purpose. pure air, nutritious food, and some preparation of iron will be invariably found useful; and those who believe its efficacy, may apply iodine locally over the thyroid gland. I have not seen complete recovery in any case, although several are recorded; but $I$ have met with considerable amelioration; and in all that I have treated, improvement has followed the steady employment of the means I suggest.

Abscess. An abscess may form within the ocular tunic, and the symptoms would be protrusion of the eyeball, and pointing or swelling externally between it and the eyelid.

Pus may be deposited in the orbita? cavity without the ocular tunic, and whether it be acute, subacute, or chronic suppuration, the physical characters will be the same; namely, the bulging of the orbital portion of the eyelid corresponding to the seat of the suppuration. 'The formation of pus is, according to my experience, a common orbital affection; and when, with protrusion, there are the usual constitutional symptoms attendant on abscess-the pain, with or without movement of the globe, the redness and puffiness of the eyelid, and the throbbing - we should early endeavour to discover the deposit by an exploratory puncture, made, if practicable, within the eyelids, in the probable direction of the abscess.

Inflammation of the Orbital Areolar Tissue, Idiopathic and Traumatic. With the protrusion from this cause, there is always much swelling and redness of the conjunctiva-chemosis, as it is called. As an idio- pathic affection in various stages, it is not uncommon. It would not answer any practical end to notice in detail the degrees of chemosis that occur; it is enough to say that in the worst cases it stands out as a vascular tumour, and thrusts the eyelids completely aside. It may be limited in extent, and confined to the upper portion of the conjunctiva. As in very slight cases there is but little protrusion of the eyeball, it may be the only objective symptom, and it is always the most marked one. There are rarely absent the pain and the constitutional disturbance.

The traumatic variety is that most frequently met with. It occurs after severe blows about the temples, or on the margin of the orbit. Of course, all the contents of this cavity are always more or less involved. With the protrusion, the eyeball is generally restricted in its movements ; sometimes it is motionless. I have been surprised to find the vision affected in all the severe cases that have come under my notice; and in all that I have been able to watch, it has never been restored.

The treatment consists in rest, leeching, or cupping at the temple, and incisions into the chemosis. It is decidedly wrong to attempt to press back the swollen conjunctiva by pulling the eyelid over it and applying a compress, as the eyelid is apt to suffer, while the disease is not thereby arrested; and it is equally improper to excise any part of the membrane, as I have seen done, for contractions follow, and entrcpium is likely to ensue. It is not necessary to dwell on general treatment.

A very rare form of this kind of protrusion is hypertrophy of the orbital areolar tissue, just as occurs in elephantiasis. In the only case I have met with, there was no loss of ocular movements. The conjunctivæ were highly injected, of a coarser structure than natural, and bolstered out around their ocular attach. ments by the posterior swellings, which were dense and doughy. Vision was perfect. There was much pain. I lost sight of the patient. I learn from the few cases which are recorded, that the disease is progressive, and the conjunctiva becoming dry and cuticular, the cornea gives way by slough or ulceration, and the eyeballs collapse. Very severe pain had induced the surgeons to resort to extirpation.

$$
\text { [To be continued.] }
$$

\section{NOTES ON HERNIA.}

By John Thompson, M.D., F.R.C.S., Bideford.

UMBILICAL hernia occurs with such frequency in infancy, that it might, without impropriety, be telmed "infantile hernia". It is sometimes congenital, but most frequently first makes its appearance some months after birth, when the muscular movements of the abdomen and lower extremities become forcible. Probably, in most cases the umbilical aperture has not been thoroughly contracted, and an expansion rather than rupture of tissue takes place. I have been struck with the comparative rarity of the complaint after the first year, which is explained on the supposition that the orifice of the unbilicus is then fully occluded.

The appearance of this hernia in infancy is peculiar, presenting a protrusion like a filbert or walnut, seldom exceeding the latter in size, except it be congenital; its delicate softness and elasticity on touch exceed these properties in the finest caoutchouc manufactured. It is not often met with in the adult; but when it occurs, is generally in females who have borne many children, and are past the middle period of life. Its size may then vary from an orange to a 
melon; and on handling, a peculiar boggy elastic feeling is communicated. An impulse is perceived in each variety of this hernia on the patient coughing or otherwise making forced expiration. I have never read of any other affection simulating this disease; and the following case, as affording an instance, may therefore interest.

A child, about five or six years of age, had suffered from fever, believed to be typhoid; but, during the convalescence, a small tumour appeared at the umbilicus, accompanied with pain, tenderness on pressure of the abdomen, and siciness.

I found, on being called in, a protrusion at the umbilicus exactly resembling umbilical hernia; it could not be returned, and was accompanier with great tenderness of the abdomen, pain, sickness, and constipation. It looked to me a case of strangulated umbilical hernia; and, as the objective and subjective symptoms were so unfavourable, relief by operation seemed a forlorn hope. In no long time, the tumour became discoloured, gangrenous, and sloughy;
and within a few days from its first appearance, gave way; but, instead of stercus, there issued pus, and this in large quantity, without admixture. The case was now explained to be abscess bursting at the umbilicus; and my fears respecting artificial anus or fæcal fistula were dissipated, as no connection with the intestine had been formed. Pus continued to issue from the opening for two or three weeks; it then ceased; the child's health became restored; he grew well; and is now a robust adult.

I believe the case was one of secondary abdominal cellulitis following fever. I have found this externally in the sheath of the rectus; and there seems no reason why it might not occur within the abdominal walls, and probably in the fascia propria; that it was not glandular abscess, seems clear from the subsequent history of the patient.

It is generally recommended to treat simple reducible umbilical hernia in the child by applying compresses of wood, lead, or lint, kept in place by plasters or bandages; or by elastic bandages with conical projections of India-rubber, or balls inflated with air, to make pressure at the umbilicus; the whole of which I deem to be very unsatisfactory. As many months, or even a year or two, may be necessary for the cure, the employment of plasters for such periods on the delicate skin of the infant, I hold to
be hardly possible, reflecting on the irritation and filth which must occur, and, moreover, that the warmth of the child's skin in this situation is continually loosening the most adhesive preparation. Ordinary bandages will shift position with the multitudinous movements of the child, notwithstanding the greatest care in their application. The wide elastic bandage, with a cone to press on the umbilicus, will sometimes fit and keep in position, but as frequently it will not; it is questionable, however, whether it be advisable to have conical pressure exerted in this disease, lest it retard the contraction of the umbilical aperture. Another objection to the elastic bandage is, that it will not wash; a matter of consequence where an article is required to be worn on the body of a young child for montls, and even years. Feeling these objections, I designed a little apparatus, which I have recommended to my patients for a great many years, and which I will briefly describe.

An abdominal support is to be made of two layers of thick calico; it is to be open behind, where some strings or a lace should be placed; the body must have several cords of stout bobbin drawn between the layers from top to bottom, to prevent folding; and, in the part which covers the umbilicus, a pocket of about two and a half inches square, opening in- wards, is to be formed, which is to receive a square of gutta percha, about the thickness of moderately stout tapping leather, with the edges pared or rounded by a file; a few stitches may then be made to keep the pocket closed. There should be shoulder- and thighstraps to keep it in place; and it then may be worn either on the outside or within the child's linen. The warmth of the abdomen moulds the gutta percha into shape, which thus effectually covers the umbilicus, and repels the hernial protrusion. It is well to have at least two abdominal supports of this kind for each patient; a soiled article can thus be replaced by a clean one, the gutta percha being removed from the one to the other. In this way, the case is managed effectively, and with due attention to comfort and cleanliness.

Obstruction, or strangulation of this hernia, may occur in the adult, as I have myself seen; but in respect to infantile umbilical hernia, I never saw, heard, or read, of a case of strangulation. A very large proportion of the cases are never placed under professional treatment; and being left to nature, with the assistance of the support afforded by the usual dress of the infant, do well. I knew a practitioner of large experience who left these cases to the care of the parents, with the assurance that they would be cured, and no untoward results were known to follow. The assistance of the surgeon may, therefore, be rather an aid than a necessity-valuable in expediting, rather than ensuring, the recovery of the patient.

\section{DISLOCATION OF THE HEAD OF THE RADIUS.}

By Henry Hare, M.D., Great Baddow.

THE following case, which occurred in my practice on September $28 \mathrm{th}$, may be considered of interest.

A boy, about 10 years of age, was thrown from a donkey, pitching on his hand. When he came to my surgery, I found the left arm hanging down by his side; the hand being in a state of pronation. I could easily bend the forearm upon the arm, and also extend it fully; and that without giving pain. I could effect supination and pronation, with the infliction of little uneasiness. 'The head of the radius could be felt on the outside of the external condyle of the humerus. It was perceived and felt to move under the finger on effecting supination and pronation. The ulna was normal in its position; and without fracture. There was no fracture of the radius. The appearance of the forearm that struck me most, was that of a depression behind, beneath the olecranon, along the margin of the ulna, making the whole of that bone prominent, brought out, as it were, in relief, when the hand was in a state of pronation. The tendon of the biceps muscle felt somewhat tense. The depression appeared less, when the hand was put in the state of supination; but did not disappear altogether. I diagnosed dislocation of the head of the radius outwards.

I made an unsuccessful attempt at reduction by extension; grasping the boy's hand with my right, and his arm with my left hand. I afterwards put my knee in the bend of the elbow, and flexed the forearm; the head of the radius immediately returned to its normal position with a snap.

When I saw the boy next morning, the arm appeared quite normal, with the exception that behind, the margin of the ulna remained somewhat prominent; and there was a slight appearance of depression (a muscular effect, I imagine) about the middle, along the margin of the ulna. 